Results There was a significant difference in SDLP3 between OSAS patients and controls $(0.44 \mathrm{v} / \mathrm{s} 0.39, \mathrm{P}=0.03) .10 \%$ of patients had worse SDLP3 than the $95^{\text {th }}$ centile among controls (Figure 1).

Conclusions Worse SDLP is a marker of poor driving performance and this is significantly worse in untreated OSAS patients as compared to controls. The choice of $95 \%$ is arbitrary but is consistent with the approach taken to establish a normal range. Establishing where a patient lies in comparison to controls may be useful in advising patients whether they are at increased risk of an accident due to OSAS. Defining a normal range based on continuously measured variable in MiniUoLDS holds promise and is a step ahead towards developing an objective test in evaluating the at risk OSAS patients.

\section{S26 IS THE "TIME SPENT WITH SATURATIONS BELOW 90\%" ON SLEEP STUDY HELPFUL IN IDENTIFYING OBESITY HYPOVENTILATION SYNDROME IN THE SLEEP CLINIC?}

GM Probert, B Prudon, SD West. Newcastle Regional Sleep Service, Newcastle-Upon-Tyne, UK

\subsection{6/thoraxjnl-2015-207770.32}

Introduction Obesity Hypoventilation Syndrome (OHS) is defined as sleep disordered breathing, obesity, and daytime hypercapnia, without another cause of ventilatory impairment. ${ }^{1}$ Recent studies have shown that a raised base excess $(\geq 2)$ or raised venous bicarbonate without daytime hypercapnia, represents a subgroup with OHS without overt respiratory failure. ${ }^{2}$ A readily available sleep study parameter indicating the presence of OHS rather than requiring biochemistry would be ideal. We assessed the use of time spent with oxygen saturations $\leq 90 \%$ from standard sleep study data and its relationship with a biochemical diagnosis of OHS.

Methods We prospectively collected data on sleep clinic patients referred for assessment of possible obstructive sleep apnoea. Patients underwent sleep studies as per standard practice, and the\%time spent with saturations $<90 \%$ was noted (more or less than $30 \%$ of the night). Venous bicarbonate or arterial blood gas was checked. Those with evidence of OHS on blood testing had assessment to exclude co-existent respiratory disease.

Results Data was collected from 190 patients, 71\% male, average age 31 (10.8, range 25-75) and mean BMI $39 \mathrm{~kg} / \mathrm{m}^{2}(8.7$, 25-76). There was biochemical evidence of OHS in 54 patients (22\%) (Venous bicarbonate $>27, \mathrm{BE} \geq 2, \mathrm{pCO}_{2} \geq 6 \mathrm{kPa}$ ). Four patients were excluded: COPD (2), Myasthenia gravis (1) and thoracic scoliosis (1).

Table 1 shows the results. Saturations of $\leq 90 \%$ for $\geq 30 \%$ of night had a sensitivity for diagnosing OHS of 59\%, specificity $47 \%$. The positive predictive value was $31 \%$ and negative predictive value was $74 \%$.

Conclusions The parameter of "time spent with saturations below 90\%" on sleep study is not particularly sensitive or specific for identifying patients with OHS in isolation. We cannot find other literature which has assessed this variable. It does not seem that it can replace blood biochemical measurement in the diagnosis of OHS. This condition still has many unanswered questions remaining including best method of diagnosis and management.
Abstract S26 Table 1 Patient numbers for those with and without $\mathrm{OHS}$, showing time spent with saturations less then $90 \%$

\begin{tabular}{|c|c|c|c|}
\hline \multicolumn{2}{|c|}{} & \multicolumn{2}{c|}{ OHS (on biochemistry) } \\
\cline { 2 - 4 } \multicolumn{2}{|c|}{} & $\begin{array}{c}\text { Saturations } \leq 90 \% \\
230 \% \text { of night }\end{array}$ & $\begin{array}{c}\text { Saturations } \leq 90 \% \\
\leq 30 \% \text { of night }\end{array}$ \\
\hline $\begin{array}{c}\text { No OHS } \\
\text { (on biochemistry) }\end{array}$ & $\begin{array}{c}\text { Saturations } \leq 90 \% \\
230 \% \text { of night }\end{array}$ & $\begin{array}{c}32 \\
\text { TRUE POSITIVE }\end{array}$ & $\begin{array}{c}22 \\
\text { FALSE NEGATIVE }\end{array}$ \\
\cline { 2 - 4 } & $\begin{array}{c}\text { Saturations } \leq 90 \% \\
\leq 30 \% \text { of night }\end{array}$ & $\begin{array}{c}72 \\
\text { FALSE POSTIVE }\end{array}$ & $\begin{array}{c}64 \\
\text { TRUE NEGATIVE }\end{array}$ \\
\hline
\end{tabular}

\section{REFERENCES}

1 Mokhlesi B, Tulaimat A. Recent advances in obesity hypoventilation syndrome. Chest 2007:132(4):1322-36

2 Manuel A, Hart N, Stradling J. Is a raised bicarbonate, without hypercapnia, part of the physiological spectrum of obesity-related hypoventilation? Thorax 2014;69 (Suppl 2): A29

\section{S27 PREDICTIVE PERFORMANCE OF STOPBANG QUESTIONNAIRE FOR DIAGNOSIS OF SLEEP APNOEA IN A CARDIAC SURGICAL COHORT}

M Mason, J Hernández-Sánchez, D Horton, A Clutterbuck-James, I Smith. Papworth Hospital NHS Foundation Trust, Cambridge, UK

\subsection{6/thoraxjnl-2015-207770.33}

Introduction and objectives Questionnaires to assess the risk of obstructive sleep apnoea (OSA) prior to surgery could reduce the need for screening sleep studies. STOPBANG questionnaire is user friendly and was previously validated in a general surgical population. A high risk of OSA has been defined as a score of $\geq 3$ and low risk as a score $0-2$. We aimed to validate the STOPBANG against nocturnal oximetry in a population undergoing major cardiac surgery and assessed its prognostic value for postoperative outcomes.

Methods Patients were screened for high risk of OSA with the STOPBANG questionnaire. The presence of sleep apnoea (SA), prior to surgery, was assessed with overnight oximetry. SA was defined as mild with a $4 \%$ oxygen desaturation index (ODI) of $5-14 / \mathrm{hr}$, moderate with ODI of $15-29 / \mathrm{hr}$ and severe ODI $\geq 30$ / hr. Predictive performance of STOPBANG against nocturnal oximetry was assessed for diagnosis of mild and moderate SA by assessing the area under curve receiver operating characteristic (AUC-ROC) and sensitivity and specificity were calculated. A multiple-logistic regression model was used to assess association of STOPBANG and post-operative outcomes.

Results The AUC-ROC for mild SA was low 0.57 (95\% CI = 0.47-0.67). Good performance was observed for moderate SA with AUC-ROC 0.82 (95\% CI $=0.69-0.95)$ (Figure 1) but specificity of STOPBANG at the conventional cut of value of $\geq 3$ for moderate SA was very low at $5 \%$ whilst sensitivity was $100 \%$. The best predictive STOPBANG cut-off value for moderate SA was $\geq 6$ with sensitivity and specificity of $75 \%$ and $77 \%$ respectively. Assessing predictive value for severe SA was not possible due to the lack of severe SA cases in our cohort. STOPBANG was not found to be an independent predictor of worse post-operative outcomes. 


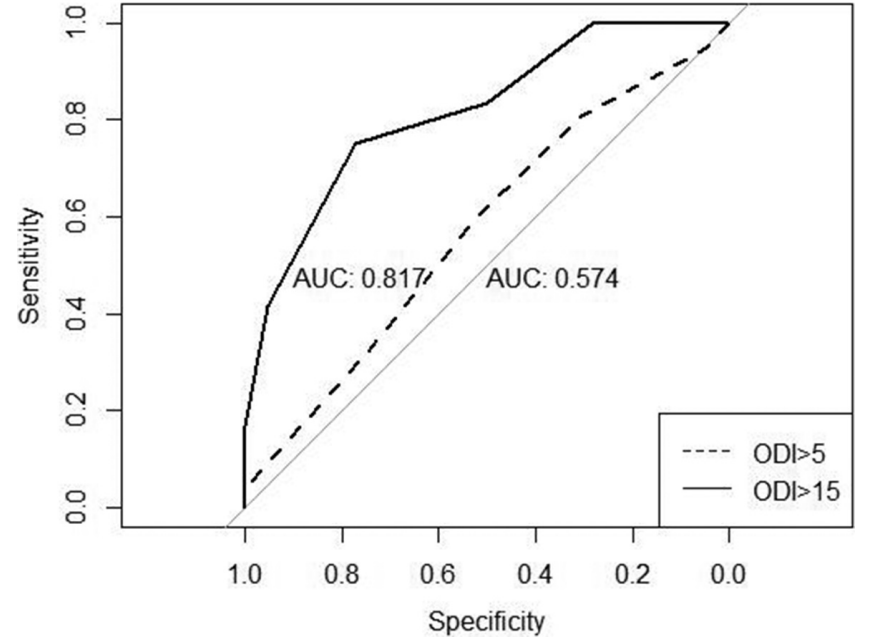

Abstract S27 Figure 1 ROC curves for STOPBANG to predict ODI $\geq 5$ and $\mathrm{ODI} \geq 15$

Conclusion Predictive performance of STOPBANG in our patient cohort at the conventional cut off value was poor. The probable explanation is that the cardiac surgical population is preselected as male, older and most suffer with hypertension. Thus the majority will score as high risk for OSA. STOPBANG had no prognostic value on worse postoperative outcomes in our study, which again contrasts with the findings in general surgical cohorts.

\section{S28 EFFECT OF SLEEP APNOEA ON POST-OPERATIVE OUTCOMES IN CARDIAC SURGERY}

M Mason, J Hernández-Sánchez, D Horton, A Clutterbuck-James, I Smith. Papworth Hospital NHS Foundation Trust, Cambridge, UK

\subsection{6/thoraxjnl-2015-207770.34}

Introduction and objectives Obstructive sleep apnoea (OSA) is common and can be associated with adverse health outcomes. There are conflicting data for the impact of undiagnosed OSA on the outcome of surgical procedures but at least some results suggest an association with worse outcomes. EuroSCORE risk model was developed to calculate the risk of mortality after cardiac surgery. We evaluated the prevalence and impact of undiagnosed sleep apnoea (SA) on postoperative outcomes in cardiac surgery.

Methods Patients undergoing coronary artery bypass grafting with or without cardiac valve surgery were screened for the presence of SA, prior to surgery, with the STOPBANG questionnaire and overnight oximetry. SA was defined as a 4\% oxygen desaturation index (ODI) of $\geq 5 / \mathrm{hr}$. A Weibull model was used to analyse lengths of stay (LoS) in intensive care unit (ICU). Complications in ICU were dichotomised and analysed with binary logistic regressions. Parsimonious models were obtained using a combination of step-wise regression and manually removing predictors that did not reach the 5\% significance level.

Results 122 subjects were included in final analysis of which 57 (47\%) had a new diagnosis of SA. Of those, 45 (79\%) had mild SA and $12(21 \%)$ had moderate/severe SA. There was no simple relationship between OSA as measured by ODI and LoS in ICU. The most significant predictor for ICU LoS was developing complications at ICU $(\mathrm{p}<0.001)$. The independent predictors associated with increasing likelihood of developing major organ complications following cardiac surgery were EuroSCORE, ODI and intravenous opioid analgesia (IOA). When patients with mild and moderate SA received IOA, predicted probability of complications rose 2.4 and 1.4 times respectively (Figure 1).

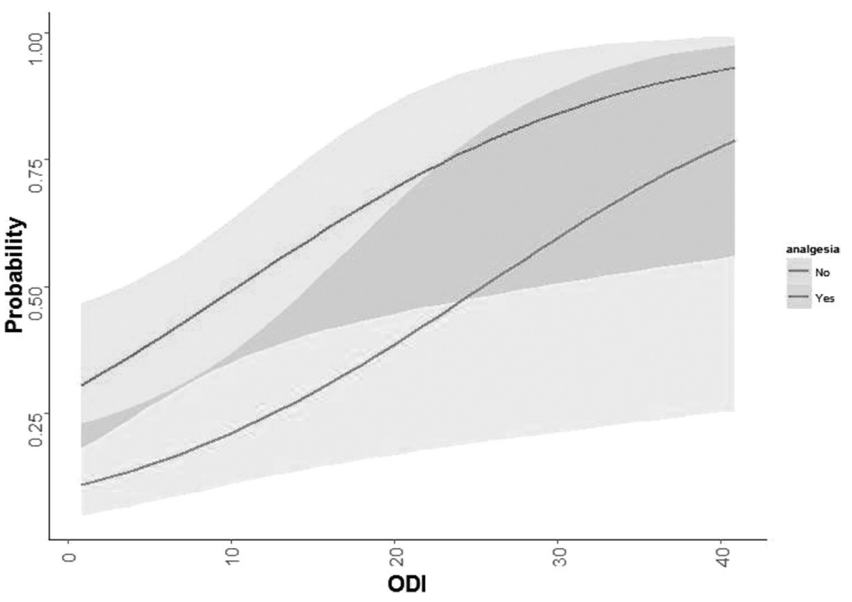

Abstract S28 Figure 1 Predicted probabilities and $95 \% \mathrm{Cl}$ of suffering a complication at ICU as ODI increases for individuals with average EuroSCORE (5) and with or without IOA

Conclusion We found a high prevalence of undiagnosed sleep apnoea in our cohort. EuroSCORE, SA and the administration of intravenous morphine were found to be independent risk factors for developing post-operative complications. This risk has increased when patients with SA received intravenous morphine.

\section{S29 PREDICTORS OF CONTINUOUS POSITIVE AIRWAYS PRESSURE USAGE AT SIX MONTHS IN MINIMALLY SYMPTOMATIC PATIENTS. FURTHER DATA FROM THE MOSAIC TRIAL}

${ }^{1} \mathrm{CD}$ Turnbull, ${ }^{2} \mathrm{DJ}$ Bratton, ${ }^{3} \mathrm{SE}$ Craig, ${ }^{2} \mathrm{M}$ Kohler, ${ }^{1} \mathrm{JR}$ Stradling. ${ }^{1}$ Oxford Centre for Respiratory Medicine and NIHR Oxford Biomedical Research Centre, Oxford, UK: ${ }^{2}$ University Hospital Zurich, Zurich, Switzerland; ${ }^{3}$ Aintree Chest Centre, Liverpool, UK

\subsection{6/thoraxjnl-2015-207770.35}

Introduction Severity of OSA and early patterns of CPAP usage have previously been shown to determine subsequent long term CPAP use in patients with symptomatic moderate-to-severe disease. ${ }^{1}$ We wished to see if different factors influenced compliance in minimally symptomatic patients.

Methods Patients were randomised to 6-months of CPAP or standard care if they had an ODI of $>7.5 \mathrm{~h}$ due to OSA on a baseline sleep study, but had insufficient daytime OSA symptoms to mandate $\mathrm{CPAP}^{2}$

Baseline characteristics (Table 1), medical history, ESS, SAQLI and SF-36 were recorded. Repeat overnight pulse oximetry was performed after entry for uniformity of trial ODI across recruiting centres. 